\title{
A RELAÇÃO ENTRE OS MODOS ADAPTATIVOS DE ROY E A TAXONOMIA DE DIAGNÓSTICOS DE ENFERMAGEM DA NANDA*
}

\author{
Marcos Venícios de Oliveira Lopes** \\ Thelma Leite de Araújo*** \\ Dafne Paiva Rodrigues****
}

LOPES, M.V.de O.; ARAÚJO, T.L.de; RODRIGUES, D.P. A relação entre os modos adaptativos de Roy e taxonomia de diagnósticos de enfermagem da NANDA. Rev.latino-am.enfermagem, Ribeirão Preto, v. 7, n. 4, p. 97-104, outubro 1999.

Estudo teórico-reflexivo objetivando estabelecer uma correlação entre a taxonomia de diagnósticos da NANDA e os modos adaptativos propostos por Roy. Foram definidas as relações entre os indicadores de adaptação positiva, os problemas comuns de adaptação propostos por Roy e os diagnósticos de enfermagem da NANDA. Os resultados nos permitiram perceber uma forte correspondência entre os conceitos existentes no modelo teórico estudado e a taxonomia de diagnósticos de enfermagem da NANDA. Concluímos que existe a possibilidade de operacionalizar o uso dos diagnósticos de enfermagem da NANDA tendo como base o processo de enfermagem fundamentado na teoria da Adaptação de Roy.

UNITERMOS: adaptação, NANDA, diagnóstico de enfermagem

\section{INTRODUÇÃO}

O Processo de Enfermagem está constituído de fases que podem variar de acordo com a teoria de Enfermagem a ser adotada. Sister Callista Roy (ROY \& ANDREWS, 1991) apresenta uma proposta de processo que inclui as seguintes fases: Avaliação de comportamento, Avaliação de estímulos, Diagnóstico de Enfermagem, Estabelecimento de Metas, Intervenção, e Avaliação.

As duas primeiras etapas constituem um levantamento de dados, considerando que este é um passo necessário para a delimitação de diagnósticos de Enfermagem. O estudo do Diagnóstico de Enfermagem, apesar de recente, motivou uma percepção mais apurada do Processo de Enfermagem, e uma melhor compreensão da sua importância.

A North American Nursing Diagnosis Association (NANDA) apud FARIAS et al. (1990, p.26) procurou conceituar Diagnóstico de Enfermagem da seguinte forma:

"Um julgamento clínico das respostas do indivíduo, da família ou da comunidade aos processos vitais ou aos problemas de saúde atuais ou potenciais, que fornece a base para a seleção das intervenções de enfermagem, para atingir resultados pelos quais o enfermeiro é responsável".

GORDON (1994) acrescenta que a definição de Diagnóstico engloba não só uma parte conceitual, apresentada anteriormente, como também uma parte estrutural que compreende três componentes: o problema de saúde, os fatores etiológicos ou relacionados, e as características definidoras ou grupo de sinais e sintomas.

O problema é o processo ou estado de saúde relacionado, manifestado pelo indivíduo, família ou comunidade. Este problema engloba variáveis bio-psicosócio-espirituais, podendo o mesmo aparecer como uma Síndrome, definida como um grupo de problemas concorrentes que tem, no mínimo, um fator etiológico comum (GORDON, 1994).

Os fatores etiológicos ou relacionados, podem ser comportamentos do cliente, elementos do ambiente, ou uma interação de ambos, e são a base para intervenções para resolver o problema. IYER et al. (1993) colocam que os fatores relacionados podem identificar fatores fisiológicos, psicológicos, sócioculturais, ambientais ou espirituais que possam estar ocasionando a reação percebida no cliente ou contribuindo para a mesma.

\footnotetext{
* Parte do Projeto Integrado "Cuidando de pessoas com alteração da pressão arterial”. CNPq ( processo no 520445/97)

** Enfermeiro, mestrando em Enfermagem pela Universidade Federal do Ceará

*** Enfermeira, Professora Adjunto do Departamento de Enfermagem da Universidade Federal do Ceará

**** Enfermeira, mestranda em Enfermagem pela Universidade Federal do Ceará
} 
As características definidoras são dados objetivos e subjetivos, sinais e sintomas que indicam a presença do diagnóstico de enfermagem. Algumas estão presentes em quase todos os clientes com o problema sendo denominadas de características críticas, cuja presença por si só determina o diagnóstico, ou maiores quando estão presentes em um grande número de clientes. As características auxiliares ajudam ou confirmam o diagnóstico e aumentam a confiança num julgamento particular (Mc FARLAND \& Mc FARLANE, 1993).

Entendemos que a necessidade de constante avaliação do Processo de Enfermagem se faz necessário, na medida em que o avançar da profissão exige a análise de seu desempenho diário, objetivando a melhoria da mesma. Assim, além do trabalho processual, o uso de teorias que visam definir a enfermagem enquanto ciência, apresenta-se como necessário para compreender questões inerentes ao cliente, ao ambiente, e à meta da enfermagem. Questões estas que são fundamentais para o desenvolvimento de um cuidado qualificado.

O modelo da Adaptação proposto por Roy (ROY \& ANDREWS, 1991) inclui a noção de estímulos e respostas. De acordo com a teórica, os estímulos subdividem-se em internos e externos. Os internos fazem parte do EU da pessoa, enquanto que os externos são incentivos ambientais.

O surgimento constante de estímulos leva a necessidade de respostas por parte do indivíduo. Para isto são acionados mecanismos de enfrentamento, que seriam modos inatos ou adquiridos de responder ao ambiente variável. Estes mecanismos de enfrentamento estão subdivididos em dois subsistemas a saber: o Subsistema Regulador que envolve os sistemas químico, neuronal e endócrino; e o Subsistema Cognoscente que envolve quatro canais cognitivos-emocionais.

Os comportamentos resultantes destes subsistemas são observados a partir de quatro modos adaptativos: a) Modo fisiológico - o modo como a pessoa responde como um ser físico aos incentivos ambientais. Este modo envolve cinco necessidades básicas de integridade fisiológica (oxigenação, nutrição, eliminação, atividade e repouso, e proteção) e quatro processos complexos (sensitivo, líquido e eletrólitos, função neurológica e função endócrina); b) Modo de autoconceito - enfoca aspectos psicológicos e espirituais da pessoa. É a combinação de convicções e sentimentos de uma pessoa num determinado momento. Inclui dois componentes: o self-físico (abrange a sensação e a autoimagem corporal) e o self-pessoal (engloba o selfconsistência, o self-ideal e o self-ético-moral-espiritual); c) Modo de desempenho de papel - enfoca aspectos sociais relacionados aos papéis que a pessoa ocupa na sociedade e d) Modo de interdependência - enfoca interações relacionadas a dar e receber afeto, respeito e valor. A necessidade básica deste modo é a afetiva, ou seja, o sentimento de segurança que permeia as relações.

Com relação ao processo de enfermagem proposto por Roy (ROY \& ANDREWS, 1991), destacase a etapa do diagnóstico, visto que, propõe uma perspectiva diferente sobre tal etapa. Para ela, a formulação do diagnóstico pode ocorrer de três forma diferentes: primeiro, uma avaliação de comportamentos de um único modo que possua muitos estímulos relevantes influenciando-o; segundo; uma classificação resumida para comportamentos em um único modo com um único estímulo relevante; e por último, uma classificação que resuma um padrão comportamental, quando mais de um modo está sendo afetado por alguns estímulos.

Foi definido ainda, uma tipologia de indicadores de adaptação positiva, que define as características das respostas tidas como eficazes em relação a um ou vários estímulos. Além disso, há também uma tipologia de problemas adaptativos de ocorrência comum que, na verdade, se apresenta como uma classificação de diagnósticos de enfermagem diferente da taxonomia da NANDA.

É importante definir a existência de diferenças entre a tipologia apresentada em ROY \& ANDREWS (1991) e a taxonomia da NANDA. Tais divergências são de caráter numérico (a tipologia apresentada por aquelas autoras relaciona uma quantidade bem menor de situações) e na forma de enunciar os diagnósticos. As autoras propõem três maneiras diferentes de fazê-lo: utilizar a referida tipologia diagnóstica; enunciar o comportamento observado acrescido dos estímulos mais influenciadores; e enunciar vários comportamentos associados com os mesmos estímulos.

Alguns obstáculos se fazem presentes na adoção deste processo diagnóstico e incluem: a pouca familiaridade com a tipologia proposta; o número reduzido de diagnósticos da tipologia; e as formas de enunciar o diagnóstico, apresentam-se extensas e não seguem o padrão proposto pela NANDA.

Devemos salientar, entretanto, que a própria tipologia de diagnósticos, presente na teoria, tem uma forte relação com a taxonomia da NANDA chegando, em alguns diagnósticos, a apresentar uma denominação semelhante. Isto pode ser fruto da participação de Callista Roy como membro da NANDA desde os primeiros trabalhos deste grupo.

\section{OBJETIVOS}

- Estabelecer uma relação entre a taxonomia de diagnósticos da NANDA e os quatro modos adaptativos propostos por Roy. 
- Relacionar os indicadores de adaptação positiva e os problemas comuns de adaptação aos diagnósticos de enfermagem da taxonomia da NANDA

\section{METODOLOGIA}

O estudo é de cunho teórico-reflexivo e envolveu um estudo aprofundado de dois tópicos: a taxonomia de diagnósticos de enfermagem da NANDA e o Modelo da Adaptação de Roy. Foram estabelecidas relações entre os indicadores de adaptação positiva, os problemas comuns de adaptação e os diagnósticos de enfermagem. A relação dos diagnósticos utilizada para o desenvolvimento da pesquisa foi a taxonomia da NANDA conforme apresentada em NÓBREGA \& GARCIA (1994), acrescentada dos diagnósticos de enfermagem aprovados pela referida associação nos anos subsequentes à obra citada. Estes diagnósticos foram encontrados em CARPENITO (1997).

Elaboramos um total de quatro quadros visando o estabelecimento de vínculos que pudessem facilitar a relação direta entre estes itens. Os passos para estabelecimento das associações foram os seguintes: primeiro, estabelecemos a semelhança conceitual entre os diagnósticos de enfermagem da NANDA e os modos adaptativos de Roy. Terminada esta primeira etapa, foi feita uma avaliação entre os problemas comuns de adaptação de cada um dos componentes dos quatro modos adaptativos propostos por Roy e os diagnósticos de enfermagem da NANDA, visando confirmar ou modificar a correspondência estabelecida no passo anterior. A última etapa tinha a finalidade de confirmar as relações propostas e baseava-se na comparação entre os fatores relacionados, características definidoras críticas, maiores e menores dos diagnósticos de enfermagem e as definições dos problemas comuns de adaptação e/ou indicadores de adaptação positiva.

A partir do estabelecimento das associações procedeu-se à análise dos quadros, visando esclarecer ao leitor os pontos críticos de cada quadro apresentado. Procuramos descrever o raciocínio empregado para a divisão dos diagnósticos, tanto em modos adaptativos, como também nos sub-componentes de cada modo, e diretamente com os problemas comuns de adaptação citados por Roy.

\section{RESULTADOS}

Quadro 1 - Relação entre os indicadores de adaptação positiva e problemas comuns de adaptação de Roy associados ao modo fisiológico, e os diagnósticos de enfermagem da NANDA - Fortaleza, 1998

\begin{tabular}{|c|c|c|}
\hline $\begin{array}{l}\text { Indicadores de adaptação positiva no } \\
\text { modo fisiológico (ROY \& ANDREWS, } \\
\text { 1991) }\end{array}$ & $\begin{array}{l}\text { Problemas comuns de adaptação no modo } \\
\text { fisiológico (ROY \& ANDREWS, 1991) }\end{array}$ & Diagnósticos de Enfermagem daNANDA \\
\hline $\begin{array}{l}\text { 1. Oxigenação } \\
\text { - Processo ventilatónio estável } \\
\text { - Padrão de troca de gases estável } \\
\text { - Transporte de gases adequado } \\
\text { - Processo de compensação adequado }\end{array}$ & $\begin{array}{l}\text { 1. Oxigenação } \\
\text { - Hipóxia/choque/Dificuldade ventilatónia } \\
\text { - Padrão de troca de gases inadequada } \\
\text { - Transporte de gases inadequado/Perfusão } \\
\text { tissular alterada } \\
\text { - Dificuldade de captação }\end{array}$ & $\begin{array}{l}\text { 1. Oxigenação } \\
\text { - Padrão respiratónio ineficaz } \\
\text { - Incapacidade para manter ventilação } \\
\text { esportânea } \\
\text { - Resposta disfuncional ao desmarne } \\
\text { ventilatóno } \\
\text { - Troca de gases prejudicada } \\
\text { - Debito cardíaco diminuído/Perfusão tissular } \\
\text { alterada } \\
\text { - Eliminação traqueobrônquica ineficaz/Risco } \\
\text { para aspiração }\end{array}$ \\
\hline $\begin{array}{l}\text { 2. Nutrição } \\
\text { - Processo de digestão estável } \\
\text { - Padrão nutricional para as necessidades } \\
\text { corporais adequado } \\
\text { - Necessidades metabólicas supridas }\end{array}$ & $\begin{array}{l}\text { 2. Nutrição } \\
\text { - Náuseas/Vômitos/Anorexia } \\
\text { - Peso } 20 / 25 \% \text { acima / Peso } 20 / 25 \% \text { abaixo i } \\
\text { Nutrição maior/me nor que as necessidades } \\
\text { corporais / Estratégias inefetivas de } \\
\text { enfrentamento para alterações na ingestão }\end{array}$ & $\begin{array}{l}\text { 2. Nutrição } \\
\text { - Deglutição prejudicada } \\
\text { - Nutrição alterada: ingestão maior do que as } \\
\text { necessidades corporais / Wutrição alterada: } \\
\text { ingestão menor do que as necessidades } \\
\text { corporais / Nutrição alterada: risco para } \\
\text { ingestão maior do que as necessidades } \\
\text { corporais / Crescimento e desenvolvimento } \\
\text { alterados / Padrão ineficaz de alimentação } \\
\text { infantil }\end{array}$ \\
\hline
\end{tabular}




\begin{tabular}{|c|c|c|}
\hline $\begin{array}{l}\text { 3. Eliminações } \\
\text { - Processos intestinais efetivos } \\
\text { - Eliminação intestinal estável } \\
\text { - Processo de formação urinária estável } \\
\text { - Padrão de eliminação urinária estável } \\
\text { - Estratégias de enfrentamento para eliminação } \\
\text { alterada eficazes }\end{array}$ & $\begin{array}{l}\text { 3. Eliminações } \\
\text { - Diarréia / Constipação / Flatulência } \\
\text { - Incontinência } \\
\text { - Oligúria } \\
\text { - Incontinência / Retenção } \\
\text { - Estratégias de enfrentamento para eliminação } \\
\text { alterada ineficazes }\end{array}$ & $\begin{array}{l}\text { 3. Eliminações } \\
\text { - Constipação / Constipação percebida / } \\
\text { Constipação colônica / Diarréia } \\
\text { - Incontinência intestinal } \\
\text { - Eliminação urinária alterada } \\
\text { - Incontinência por pressão / Incontinência } \\
\text { reflexa / Incontinência impulsiva / } \\
\text { Incontinência funcional / Incontinência total } \\
\text { / Retenção urinária }\end{array}$ \\
\hline $\begin{array}{l}\text { 4. Atividade e Repouso } \\
\text { - Processo de mobilidade integrado } \\
\text { - Processo compensatório de movimentação } \\
\text { durante a inatividade adequado } \\
\text { - Padrão de atividade e repouso efetivo / Padrão } \\
\text { de sono efetivo / Mudanças ambientais } \\
\text { influenciam nas condições de sono }\end{array}$ & $\begin{array}{l}\text { 4. Atividade e Repouso } \\
\text { - Processo de mobilidade restrito / Dificuldade } \\
\text { para deambular / Incoordenação / Imobilidade } \\
\text { - Intolerância à atividade / Conseqüências de } \\
\text { desuso } \\
\text { - Risco para distúrbio no padrão de sono / } \\
\text { Privação de sono / Fadiga / Mudanças } \\
\text { ambientais não influenciam nas condições de } \\
\text { sono }\end{array}$ & $\begin{array}{l}\text { 4. Atividade e Repouso } \\
\text { - Risco para trauma por posicionamento } \\
\text { perioperatório / Risco para disfunção } \\
\text { neurovascular periférica } \\
\text { - Risco para intolerância à atividade / } \\
\text { Intolerância à atividade / Risco para } \\
\text { síndrome do desuso / Mobilidade física } \\
\text { prejudicada } \\
\text { - Distúrbio no padrão de sono / Fadiga }\end{array}$ \\
\hline $\begin{array}{l}\text { 5. Proteção } \\
\text { - Pele íntegra } \\
\text { - Processos imunitários efetivos } \\
\text { - Cicatrização efetiva } \\
\text { - Proteção secundária para mudanças na } \\
\text { integridade da pele e no status imune adequada }\end{array}$ & $\begin{array}{l}\text { 5. Proteção } \\
\text { - Lesão / Prurido / Hematoma } \\
\text { - Alergias / Déficit imunitário / Potencial para } \\
\text { enfrentamento inefetivo de reação alérgica } \\
\text { - Cicatrização comprometida } \\
\text { - Proteção secundária para mudanças na } \\
\text { integridade da pele e no status imune ineficaz / } \\
\text { Infecção }\end{array}$ & $\begin{array}{l}\text { 5. Proteção } \\
\text { - Integridade da pele prejudicada } \\
\text { - Risco para infecção } \\
\text { - Mucosa oral alterada } \\
\text { - Proteção alterada }\end{array}$ \\
\hline $\begin{array}{l}\text { 6. Sentidos } \\
\text { - Processo de sensação efetivo } \\
\text { - Integração sensorial para recebimento de } \\
\text { informações efetiva } \\
\text { - Padrões de recepção, interpretação, e } \\
\text { apreciação de estímulos estáveis } \\
\text { - Estratégias de enfrentamento para alterações } \\
\text { dos sentidos efetivas }\end{array}$ & $\begin{array}{l}\text { 6. Sentidos } \\
\text { - Deficiência de um sentido primário / } \\
\text { Deficiência perceptiva / Estigma } \\
\text { - Deficiência de integração sensorial / Risco } \\
\text { para comunicação distorcida } \\
\text { - Diminuição na habilidade para o autocuidado/ } \\
\text { Potencial para injúria / Distorção sensorial / } \\
\text { Monotonia sensorial / Privação sensorial / } \\
\text { Excesso de sensório / Dor aguda / Dor crônica/ } \\
\text { Estratégias de enfrentamento ineficazes para } \\
\text { deficiência sensorial }\end{array}$ & $\begin{array}{l}\text { 6. Sentidos } \\
\text { - Sensopercepção alterada / Percepção } \\
\text { unilateral alterada } \\
\text { - Comunicação prejudicada } \\
\text { - Déficit no autocuidado: alimentação, } \\
\text { higiene corporal, vestir-se e/ou arrumar-se, } \\
\text { higiene íntima } \\
\text { - Dor aguda / Dor crônica / Risco para } \\
\text { injúria/ Risco para envenenamento / Risco } \\
\text { para trauma }\end{array}$ \\
\hline $\begin{array}{l}\text { 7. Líquidos e eletrólitos } \\
\text { - Balanço hídrico estável } \\
\text { - Sais nos fluídos corpóreos estáveis } \\
\text { - Status ácido / base equilibrado } \\
\text { - Regulação química estável }\end{array}$ & $\begin{array}{l}\text { 7. Líquidos e eletrólitos } \\
\text { - Desidratação / Edema / Choque } \\
\text { - Retenção intracelular de água / Hipocalemia / } \\
\text { Hipocalcemia / Hiponatremia } \\
\text { - Alcalose / Acidose } \\
\text { - Regulação química instável }\end{array}$ & $\begin{array}{l}\text { 7. Líquidos e eletrólitos } \\
\text { - Excesso de volume de líquidos } \\
\text { - Risco para déficit de volume de líquidos } \\
\text { - Déficit de volume de líquidos }\end{array}$ \\
\hline $\begin{array}{l}\text { 8. Função neurológica } \\
\text { - Processo de atenção / vigília efetivo } \\
\text { - Sensação / percepção; codificação, formação } \\
\text { de conceitos, memória, linguagem, resposta } \\
\text { motora eficazes } \\
\text { - Processo de pensamento e de sentidos } \\
\text { íntegros } \\
\text { - Desenvolvimento funcional e plástico, } \\
\text { envelhecimento, e alteração do sistema } \\
\text { Nervoso efetivo }\end{array}$ & $\begin{array}{l}\text { 8. Função neurológica } \\
\text { - Diminuição do nível de consciência } \\
\text { - Diminuição do processo cognitivo / Déficit } \\
\text { de memória / Compensação ineficaz para déficit } \\
\text { cognitivo } \\
\text { - Comportamento instável } \\
\text { - Risco para dano cerebral secundário }\end{array}$ & $\begin{array}{l}\text { 8. Função neurológica } \\
\text { - Risco para sufocação } \\
\text { - Capacidade adaptativa intracraniana } \\
\text { diminuída } \\
\text { - Manutenção da saúde alterada / Déficit de } \\
\text { conhecimento / Síndrome de interpretação } \\
\text { ambiental prejudicada } \\
\text { - Processo de pensamento alterado / } \\
\text { Comportamento infantil desorganizado / } \\
\text { Risco para comportamento infantil } \\
\text { desorganizado / Comportamento infantil: } \\
\text { potencial para melhoria da organização / } \\
\text { Disreflexia } \\
\text { - Termorregulação ineficaz / Hipotermia / } \\
\text { Hipertemia / Risco para temperatura corporal } \\
\text { elevada }\end{array}$ \\
\hline
\end{tabular}




\begin{tabular}{|l|l|l|}
\hline 9. Função endócrina & 9. Função endócrina & 9. Função endócrina \\
- Regulação homonal de processos corporais & - Inefe tiva regulação hormonal refle tida em & - Padrão de sexualidade alterado \\
e metabólicos efetiva & fadiga, imitabilidade, intolerância ao calor & - Amamentação ineficaz \\
- Deservolvimento reprodutivo efetivo & - Desenvolvimento reprodutivo ineficaz & - Amamentação eficaz \\
- Estabilidade de integração do sistema & - Integração do sistema hormonal instável & - Amamentação interrompida \\
hormonal & & - Desgaste da pessoa que presta o cuidado \\
- Ritmo hormonal cíclico estável & - Ritmo hormonal cíclico instável & - Síndrome de estresse de mudança do \\
- Estratégias de enfrentamento do estresse & - Estresse & ambiente \\
efetivas & &
\end{tabular}

O primeiro quadro propõe a relação entre os indicadores de adaptação, os problemas comuns de adaptação do modo fisiológico colocados por ROY \& ANDREWS (1991), e os diagnósticos de enfermagem da taxonomia da NANDA conforme apresentada em NÓBREGA \& GARCIA (1994). A partir dessa relação percebemos que há uma quantidade muito grande de diagnósticos associados a este modo. Mais que isso, parece haver uma ligação muito ampla entre o que Roy chama de problemas comuns de adaptação e os títulos e conceitos dos diagnósticos de enfermagem da NANDA.

A função neurológica, especificamente, configura como um dos componentes de mais difícil análise. Este fato pode ser devido à condição de relação entre este processo complexo e os demais componentes do modo fisiológico. Um exemplo disso é a forte relação entre os diagnósticos termorregulação ineficaz, hipotermia, hipertermia, risco para temperatura corporal elevada, e o componente proteção. O raciocínio poderia nos levar a classificar tais diagnósticos no referido modo, já que mudanças na temperatura corporal estão muito associadas ao surgimento de infecções. Porém, a presença destes diagnósticos no componente proteção, estaria muito mais associada a uma questão de sinais, do que propriamente um diagnóstico. Melhor exemplificando, a mudança da temperatura corporal poderia ser uma conseqüência da presença de outro diagnóstico, como por exemplo a proteção alterada. Além do mais, a mudança da temperatura corporal é antes de tudo, uma função dependente do estímulo/funcionamento do sistema neurológico.

Os componentes líquidos e eletrólitos e a função endócrina tem uma característica em comum, que é uma quantidade de diagnósticos muito menor que os indicadores de adaptação e problemas comuns de adaptação. Os líquidos e eletrólitos, em princípio determinaram diagnósticos que associavam-se apenas à quantidade de líquidos corporais embora, segundo Roy, os problemas comuns de adaptação incluam também a variação de eletrólitos. De fato, na taxonomia da NANDA não existem diagnósticos que se refiram a tais variações, existem sim, diagnósticos que apresentam a variação de eletrólitos como características definidoras dos mesmos. Isto talvez, pelo fato de que os indícios de mudanças de eletrólitos sejam primeiramente refletidos nos líquidos intra e extra-celulares.

A função endócrina passa por uma relação semelhante à função neurológica, ou seja, muitos diagnósticos têm uma associação conceitual mais próxima de outros componentes e/ou até mesmo outros modos, de forma que a função endócrina pode contribuir para o estabelecimento de diversos diagnósticos em outros componentes. Um exemplo disso é o diagnóstico disfunção sexual que tem relação com o modo de autoconceito, especificamente a sub-área self-físico. Devido a estreita relação da função endócrina com os outros componentes do modo fisiológico, pode-se considerar que alguns diagnósticos mencionados em outros componentes podem vir a ser classificados na função endócrina como uma conseqüência de distúrbios específicos, como por exemplo: Hipertermia, Hipotermia, Risco para temperatura corporal elevada, Nutrição alterada: ingestão maior/menor do que as necessidades corporais, Alterações no volume de líquidos (excesso, déficit, risco para déficit) e alterações intestinais (Constipação e Diarréia).

Quadro 2 - Relação entre os indicadores de adaptação positiva e problemas comuns de adaptação de Roy associados ao modo de autoconceito, e os diagnósticos de enfermagem da NANDA - Fortaleza, 1998

\begin{tabular}{|c|c|c|}
\hline $\begin{array}{l}\text { Indicadores de adaptação positiva no } \\
\text { modo de autoconceito (ROY \& } \\
\text { ANDREWS, 1991) }\end{array}$ & $\begin{array}{l}\text { Problemas comuns de adaptação no } \\
\text { modo de autoconceito (ROY \& } \\
\text { ANDREWS, 1991) }\end{array}$ & $\begin{array}{l}\text { Diagnósticos de Enfermagem da } \\
\text { NANDA }\end{array}$ \\
\hline $\begin{array}{l}\text { 1. Selffisico } \\
\text { - Imagem corporal positiva } \\
\text { - Função sexual positiva } \\
\text { - Integridade psíquica em acordo com } \\
\text { deservolvimento fisico } \\
\text { - Compensação adequada para mudanças } \\
\text { corporais } \\
\text { - Estratégias de enfientamento efetivas paraperda } \\
\text { - Processos efetivos de fim de vida }\end{array}$ & $\begin{array}{l}\text { 1. Selffisico } \\
\text { - Distúrbio da imagem corporal } \\
\text { - Disfunção sexual } \\
\text { - Síndrome do traurna de estupro } \\
\text { - Perda }\end{array}$ & $\begin{array}{l}\text { 1. Selffisico } \\
\text { - Distúrbio da irnagem corporal } \\
\text { - Disfunção sexual } \\
\text { - Síndrome do traumade estupro / Síndrome } \\
\text { do trauma de estupro: reação composta / } \\
\text { Síndrome do trauma de est upro: reação } \\
\text { silenciosa }\end{array}$ \\
\hline
\end{tabular}




\begin{tabular}{|c|c|c|}
\hline $\begin{array}{l}\text { 2. Self-pessoal } \\
\text { - Padrão estável de autoconsistência } \\
\text { - Integração efetiva do self-ideal } \\
\text { - Processos efetivos de deservolvimento ético } \\
\text { - moral - espiritual } \\
\text { - Auto-estima funcionante } \\
\text { - Estratégias de enfrentamento efetivas para } \\
\text { ameaças do self }\end{array}$ & $\begin{array}{l}\text { 2. Selfpessoal } \\
\text { - Ansiedade } \\
\text { - Impotência } \\
\text { - Culpa } \\
\text { - Auto-estima baixa }\end{array}$ & $\begin{array}{l}\text { 2. Self-pessoal } \\
\text { - Ansiedade / Adaptação prejudicada / } \\
\text { Comportamento para elevar núvel de saúde } \\
\text { - Impotência / Desesperança } \\
\text { - Conflito de decisão } \\
\text { - Distúrbio da auto-estima / Baixa auto-estima } \\
\text { crônica / Baixa auto-estima situacional } \\
\text { - Estratégias ineficazes de resolução individual } \\
\text { / Estratégias defensivas de resolução individual } \\
\text { / Negação / Recusa / Distúrbio da identidade } \\
\text { pessoal / Medo / Resposta pós-trauma / } \\
\text { Controle ineficaz do regime terapêutico } \\
\text { individual }\end{array}$ \\
\hline
\end{tabular}

O modo de autoconceito apresenta uma certa relação com o modo fisiológico, visto que alterações de cunho biológico afetam, com uma certa freqüência, o julgamento que a pessoa tem de si mesma. Neste âmbito, a sub-área self-físico, apresenta uma relação mais direta com conceitos biológicos. É o caso dos diagnósticos Disfunção sexual e do Padrão de sexualidade alterada que podem apresentar características definidoras de cunho físico, porém, o comportamento associado emerge como conceitos pessoais de insatisfação.

Vale ressaltar que existe forte relação entre os diagnósticos acima discutidos e a função endócrina do modo fisiológico, de forma que seria aceitável uma classificação em que estes diagnósticos fossem colocados neste modo. Particularmente, preferimos a associação com o self-físico do modo de autoconceito, por entendermos que estes diagnósticos apresentam comportamentos determinados por conceitos físicos que a pessoa apresenta de sua função sexual, de forma a interferir diretamente no sub-componente sensação corporal da sub-área do self-físico de Roy, que determina a habilidade para sentir-se e experienciar-se como um ser físico.

O self-pessoal engloba aspectos de auto consistência, valores éticos, morais e espirituais, além de sentimentos de aspiração pessoal. Desta forma, os diagnósticos aqui relacionados, abrangem não somente problemas de adaptação, mas também indicadores de adaptação positiva, como é o caso do diagnóstico Comportamento para elevar o nível de saúde. Além disso, alguns diagnósticos apresentam uma relação mais ampla, podendo abranger não apenas um problema de adaptação, mas um conjunto de problemas, determinando estratégias ineficazes de enfrentamento para ameaças do self. Neste caso, relacionamos sete diagnósticos, que incluem desde Resolução ineficaz de problemas até Recusa. Este último se faz interessante discutir, na medida em que o diagnóstico Recusa apesar de ser definido como a resolução deliberada de não adesão à recomendação terapêutica, significa também, uma forma ineficaz de enfrentamento para uma ameaça ao self, representada pelo distúrbio que produziu a necessidade terapêutica.

Quadro 3 - Relação entre os indicadores de adaptação positiva e problemas comuns de adaptação de Roy associados ao modo de desempenho de papéis, e os diagnósticos de enfermagem da NANDA - Fortaleza, 1998

\begin{tabular}{|c|c|c|}
\hline $\begin{array}{l}\text { Indicadores de adaptação positiva nomodo } \\
\text { de desempenho de papel (ROY \& } \\
\text { ANDREWS, 1991) }\end{array}$ & $\begin{array}{l}\text { Problemas comuns de adaptação no } \\
\text { modo de desempenho de papel(ROY } \\
\text { \& ANDREWS, 1991) }\end{array}$ & Diagnósticos de Enfermagem da NANDA \\
\hline $\begin{array}{l}\text { - Processos efetivos de transição de papel } \\
\text { - Integração entre o instrumental e os } \\
\text { comportamentos expressos no papel } \\
\text { - Integração entre papés primánios, secundánios } \\
\text { e terciánios } \\
\text { - Padrão estável de papel dominante } \\
\text { - Processo efetivos para enfrentamento de } \\
\text { mudarças de papéis }\end{array}$ & $\begin{array}{l}\text { - Conflitos de papéis } \\
\text { - Falha no papel }\end{array}$ & 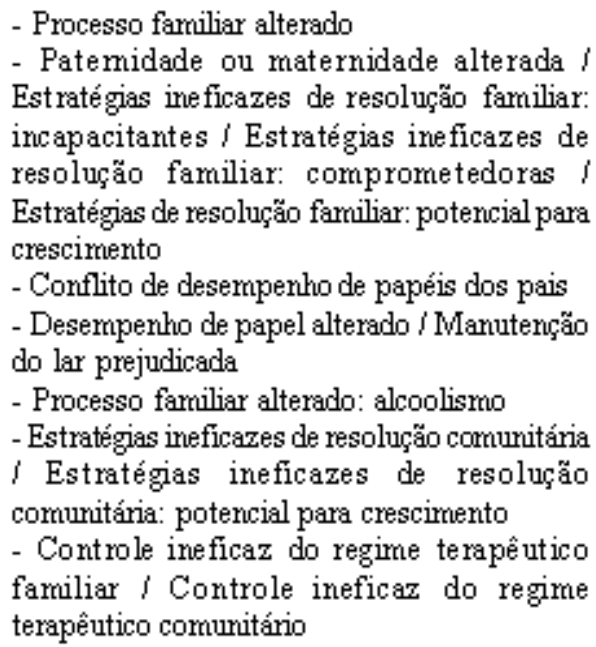 \\
\hline
\end{tabular}


Com relação ao quadro 3, os quatro itens que representam problemas de adaptação ao desempenho de papéis, foram associados a diagnósticos de enfermagem da taxonomia da NANDA, tendo como base a análise conceitual dos mesmos. Assim, o problema de distanciamento do papel determina diagnósticos que representam um comportamento que não corresponde àquilo que é esperado do sujeito.

Os diagnósticos associados a estratégias ineficazes de resolução denotam uma incapacidade de adaptar-se a uma nova realidade e de assumir um comportamento condizente com o esperado. As características definidoras de tais diagnósticos incluem atitudes de desamparo e dependência total, o que de fato, impede o estabelecimento de uma resposta adaptativa.

A transição de papel inclui uma resposta associada a um momento em que se está processando mudanças nos papéis que a pessoa está desempenhando.
Tais mudanças exigem que a própria pessoa as acompanhe, interagindo e adaptando-se. Desta forma, o Processo familiar alterado define uma disfunção experimentada por uma família e relacionada a situações de transição ou crise, determinando uma forte relação com o problema de adaptação proposto por Roy.

Com relação ao problema de falha no papel, os diagnósticos associados conduzem a situações em que, na verdade, não existe uma mudança no papel a ser desempenhado, e sim mudanças na forma de desempenhálo, de modo a produzir ambigüidades ou até mesmo distúrbios na forma de conduzir seu papel. Os diagnósticos de Desempenho de papel alterado e Manutenção do lar prejudicada apresentam fatores relacionados que incluem a ausência de modelo de papel, organização ou planejamento insuficientes, que indicam uma resposta ineficaz às exigências estabelecidas pelo papel.

Quadro 4 - Relação entre os indicadores de adaptação positiva e problemas comuns de adaptação de Roy associados ao modo de interdependência, e os diagnósticos de enfermagem da NANDA - Fortaleza, 1998

\begin{tabular}{|c|c|c|}
\hline $\begin{array}{l}\text { Indicadores de adaptação positiva no } \\
\text { modo de interdependência (ROY \& } \\
\text { ANDREWS, 1991) }\end{array}$ & $\begin{array}{l}\text { Problemas comuns de adaptação no } \\
\text { modo de interdependência (ROY \& } \\
\text { ANDREWS, 1991) }\end{array}$ & Diagnósticos de Enfermagem da NANDA \\
\hline $\begin{array}{l}\text { - Padrão estável de dar e receber energia l } \\
\text { Adequação afetiva } \\
\text { - Padrões efetivos de isolamento e } \\
\text { relacionamento } \\
\text { - Estratégias de enfrentamento efetivas para } \\
\text { separação e solidão }\end{array}$ & $\begin{array}{l}\text { - Padrão inefetivo de dar e receber energia } \\
\text { - Padrões inefetivos de isolamento e } \\
\text { relacionamento } \\
\text { - Ansiedade de separação } \\
\text { - Solidão }\end{array}$ & $\begin{array}{l}\text { - Distúrbio do campo energético } \\
\text { - Risco para violência direcionada a si e aos } \\
\text { outros } \\
\text { - Risco para mutilação } \\
\text { - Isolamento social / Interação social } \\
\text { prejudicada } \\
\text { - Risco para vínculo pais - fillhos alterado } \\
\text { - Disfunção da reação de pesar / Reação de } \\
\text { pesar antecipada / Angústia espintual } \\
\text { - Risco para solidão } \\
\text { - Potencial para melhoria do berm-estar } \\
\text { espiritual }\end{array}$ \\
\hline
\end{tabular}

O modo de interdependência apresenta conceitos associados à necessidade de dar e receber afeto, de modo que, na taxonomia da NANDA encontramos diagnósticos de enfermagem em três padrões diferentes (Sentir, Relacionar e Valorizar) que definem maneiras de responder afetivamente a estímulos ambientais. O padrão ineficaz de dar e receber energia, é bem visualizado ao se atentar para o diagnóstico de risco para violência, no qual o indivíduo apresenta comportamentos que podem ser dolorosos para si e para os outros, demonstrando uma instabilidade em seus sistemas de apoio.

Um outro problema comum de adaptação proposto por Roy (ROY \& ANDREWS, 1991) são os padrões ineficazes de isolamento e relacionamento que conduzem aos diagnósticos de enfermagem Isolamento social e Interação social prejudicada, alocados no padrão Relacionar da taxonomia da NANDA, e que definem interações ineficazes com outros sujeitos. Os três últimos diagnósticos associados ao modo de interdependência, são reações cujo estímulo focal é determinado pela separação ou possibilidade de separação de coisas significativas para a pessoa. Em geral, estes diagnósticos têm forte correlação com o conceito de perda.

\section{CONSIDERAÇÕES FINAIS}

A relação aqui apresentada entre os modos adaptativos de Roy e a taxonomia de diagnósticos de enfermagem da NANDA, não é uma proposta acabada. Na verdade, configura uma tentativa de contribuição para o uso do referencial teórico da adaptação numa forma mais próxima das tendências atuais, no que diz respeito ao uso de diagnósticos de enfermagem. É possível que possam existir propostas diferentes de correlação, fato explicável pela tentativa dos estudiosos do assunto em 
estabelecer vínculos entre os modelos teóricos existentes e a taxonomia da NANDA, já que esta última foi elaborada sem um vínculo direto com tais modelos.

$\mathrm{O}$ estabelecimento da relação proposta exigiu uma análise aprofundada de conceitos, tendo em vista o fato de o modelo teórico adotado apresentar uma forma diferente de processar e classificar os diagnósticos de enfermagem. Assim, entendemos que os parâmetros para o estabelecimento de uma relação como a que aqui propusemos, englobam especificamente a análise conceitual do diagnóstico e dos modos adaptativos, além de uma consulta aos fatores relacionados tendo em vista a complexidade e interrelação entre os modos adaptativos que compõem a pessoa.

\section{THE RELATIONSHIP AMONG THE ADAPTATION MODES OF ROY AND NANDA TAXONOMY OF NURSING DIAGNOSES}

The present study is theoretical-reflexive with the purpose to establish a correlation between NANDA's taxonomy diagnoses and the adaptation modes proposed by Roy. Authors defined the relationships among the indicators of positive adaptation, the common problems of adaptation proposed by Roy and NANDA's nursing diagnoses. Results showed a strong correspondence between the existent concepts in the studied theoretical model and NANDA's taxonomy of nursing diagnoses. Authors concluded that there is a possibility of using NANDA's nursing diagnoses and the nursing process based on Roy's Adaptation Theory.

KEY WORDS: adaptation, NANDA, nursing diagnosis

\section{LA RELACIÓN ENTRE LOS MODOS ADAPTATIVOS DE ROY Y LA TAXONOMÍA DE DIAGNÓSTICOS DE ENFERMERÍA DE LA NANDA}

Estudio teórico-reflexivo buscando establecer una correlación entre los diagnósticos de enfermería de la NANDA y los modos adaptativos propuestos por Roy. Fueron definidas las relaciones entre los indicadores de adaptación positiva, los problemas comunes de adaptación propuestos por Roy y los diagnósticos de enfermería de la NANDA. Los resultados permitieron ver una correlación fuerte entre los conceptos existentes en el modelo teórico estudiado y la taxonomía de la NANDA de diagnósticos de enfermería. Nosotros concluimos que existe la posibilidad de operacionalizar el uso de los diagnósticos de enfermería de la NANDA teniendo como base el proceso de enfermería basado en la teoría de la Adaptación de Roy.

TÉRMINOS CLAVES: adaptación, NANDA, diagnosticos de enfermería

\section{REFERÊNCIAS BIBLIOGRÁFICAS}

01. CARPENITO, L.J. Diagnósticos de enfermagem: aplicação à prática clínica. 6. ed. Porto Alegre: Artes Médicas, 1997. 812p.

02. FARIAS, J.N. et al. Diagnóstico de enfermagem: uma abordagem conceitual e prática. João Pessoa: Santa Marta, 1990.

03. GORDON, M. Nursing diagnosis: process and application. 3. ed. St Louis: Mosby, 1994. 421p.

04. IYER, P.W. et al. Processo e diagnóstico em enfermagem. Porto Alegre: Artes Médicas, 1993. 325p.
05. Mc FARLAND, G.K.; Mc FARLANE, E.A. Nursing diagnosis e intervention: planning for patient care. 2. ed. St Louis: Mosby, 1993. 811p.

06. NÓBREGA, M.M.L.; GARCIA, T.R. Uniformização da linguagem dos diagnósticos de enfermagem da NANDA: sistematização das propostas do II SNDE. João Pessoa: Grupo de Interesse em Diagnóstico de Enfermagem da Paraíba - GIDE, Comitê Nacional de Revisão de Diagnósticos de Enfermagem - CNRDE, 1994. 65p.

07. ROY, S.C.; ANDREWS, H.A. The Roy adaptation model: the definitive statement. Norwalk, Connecticut: Appleton e Lange, 1991. 472p. 